\title{
Coping strategies among caregivers of people with Alzheimer disease: a systematic review
}

\author{
Estratégias de coping entre cuidadores de pessoas com doença de Alzheimer: \\ uma revisão sistemática
}

\begin{abstract}
Alexandre Magno Frota Monteiro, Raquel Luiza Santos, Nathália Kimura, Maria Alice Tourinho Baptista,
\end{abstract} Marcia Cristina Nascimento Dourado

\begin{abstract}
Introduction: Caregivers of people with Alzheimer disease (PWAD) report significant stress, burden and depression compared to caregivers of people with other dementias, especially when neuropsychiatric symptoms are prominent. Adequate coping strategies can modify the impact of stressful situations and increase the caregivers' quality of life.

Objective: To systematically review the different coping strategies used by caregivers of PWAD to manage neuropsychiatric symptoms.

Method: We carried out electronic searches using MEDLINE (PubMed), SciELO, Web of Knowledge Cross Search (Thomson Scientific/ISI Web Services) and PsycINFO databases to select studies on coping in PwAD caregivers published from January 2005 to July 2017. The search terms were coping, caregivers, strategy, onset, adaptation, family, behavior, dementia and Alzheimer. The studies were organized in three categories: problem-focused, emotion-focused and dysfunctional coping strategies.

Results: We found 2,277 articles. After application of exclusion criteria and exclusion of redundant references, 24 articles were analyzed. Emotion-focused coping was the most commonly used strategy among PwAD caregivers. The use of this strategy associated with religion and spirituality may help reduce symptoms of depression and anxiety. Problem-focused coping strategies were mostly used with active coping interventions. Problem-solving coping may have buffered the impact of acute psychological stressors on procoagulant activity. Dysfunctional coping strategies were associated with increase of caregiver burden.

Conclusion: The evaluated studies showed that the use and development of coping strategies may have ameliorated the depressive symptoms, anxiety and burden of caregivers. However, longitudinal studies are still needed that clearly describe the type of coping strategy used in relation to the presented results.
\end{abstract}

Keywords: Alzheimer disease, coping strategies, depression, caregivers.

\section{Resumo}

Introdução: Cuidadores de pessoas com doença de Alzheimer relatam mais estresse, sobrecarga e depressão em comparação com cuidadores de pessoas com outras patologias, especialmente quando os sintomas neuropsiquiátricos são proeminentes. Estratégias adequadas de enfrentamento podem modificar o impacto de situações estressantes e aumentar a qualidade de vida do cuidador.

Objetivo: Revisar sistematicamente as diferentes estratégias de enfrentamento utilizadas pelos cuidadores para lidar com sintomas neuropsiquiátricos.

Método: Foi realizada busca eletrônica em artigos publicados entre janeiro de 2005 e julho de 2017 nos bancos de dados MEDLINE (PubMed), SciELO, Web of Knowledge Cross Search (Thomson Scientific/ISI Web Services) e PsycINFO. Os termos utilizados foram coping, caregivers, strategy, onset, adaptation, family, behavior, dementia and Alzheimer. Os artigos avaliados foram categorizados em estratégias de enfrentamento focadas na resolução do problema, na emoção e estratégias disfuncionais.

Resultados: Foram encontrados 2.277 artigos. Após aplicação dos critérios de exclusão, foram incluídos 24 artigos. A estratégia de enfrentamento mais utilizada foi a focada na emoção. O uso desta estratégia associada a religião e espiritualidade pode reduzir sintomas de depressão e ansiedade dos cuidadores. As estratégias de enfrentamento focadas na resolução do problema utilizaram principalmente intervenções baseadas no enfrentamento ativo. A resolução de problemas reduz o impacto dos estressores psicológicos agudos sobre a atividade procoagulante. As estratégias disfuncionais estavam associadas ao aumento da sobrecarga dos cuidadores.

Conclusão: Os estudos mostraram que o desenvolvimento e uso de estratégias de enfrentamento pode aliviar sintomas depressivos, ansiosos e sobrecarga do cuidador. No entanto, ainda são necessários estudos longitudinais que descrevam claramente o tipo de estratégia de enfrentamento utilizada em relação aos desfechos das pesquisas.

Descritores: Doença de Alzheimer, estratégias de enfrentamento, depressão, cuidadores. 


\section{Introduction}

The stress and burden associated with caring for people with Alzheimer disease (PWAD) not only affects the health of caregivers and increases their mortality risk, but also reduces the quality of caregiving. ${ }^{1,2}$ Caregivers of PWAD report more stress, burden and depression compared to caregivers of people with other dementias. ${ }^{3}$ Coping may modify the impact of stressful situations on quality of life.

Coping strategies are defined as specific behavioral and psychological efforts to handle or minimize stressful events. ${ }^{4}$ Lazarus et al. ${ }^{5}$ defined coping as "the constant cognitive change and behavioral adaptation when handling specific external and/or internal demands that are evaluated as something that exceeds the resources of the person." Coping is a dynamic process, as it consists of a series of reciprocal responses through which the individual and the environment interact and influence each other, and it includes a series of intentional actions, both cognitive and behavioral, meant to control the negative impact of the stressful event or situation.

Managing stressful situations can include minimizing, avoiding, tolerating, and accepting the stressful conditions, as well as attempting to master the environment. ${ }^{5}$ Coping strategies may be an important and theoretically modifiable determinant of psychological morbidity. ${ }^{5}$ Therefore, they include all the cognitive, emotional and behavioral measures adopted by an individual in response to specific internal and/or external demands that are deemed to exceed his or her normal resources. ${ }^{5}$

The model of coping involves a connection between internal and external elements: challenges and threats (internal or external, e.g., sickness or an earthquake) and personal resources (internal and external, e.g., high perceived self-efficacy and resource-rich social environment) are viewed as interrelated factors. ${ }^{6}$ Coping behaviors can be divided into three categories: problem-focused (practical steps to remove or reduce the stressor), emotion-focused (managing one's emotional responses to stress), and dysfunctional (disengaging from the stressful situation or emotions). ${ }^{7}$

Studies have shown controversial results about the more efficient coping strategies for caregivers of PwAD. A longitudinal study found that problem-focused and emotion-focused coping mediated an effect between primary stressors (associated with PwAD care needs, including their personal characteristics and illness/ disability) and decreased caregiver depression a year later. $^{8}$ In addition, Vitaliano et al. ${ }^{9}$ suggested that emotion-focused, but not problem-focused, strategies predicted caregiver burden 15-18 months later.
Conversely, Vedhara et al. ${ }^{10}$ reported that dysfunctional, but not emotion or problem-focused, coping strategies predicted greater anxiety and depression on a nonstandard measure 6-12 months later.

Understanding the different types of coping strategies used by caregivers of PWAD may increase the quality of life of both PWAD and their caregivers, as it allows the development of specific and adequate intervention strategies focused on this group. Thus, this systematic review aims to evaluate the different categories of coping strategies frequently used, as well as the effect of these strategies on the quality of life of PWAD and their caregivers.

\section{Method}

This systematic review used the PRISMA (Preferred Reporting Items for Systematic Reviews and MetaAnalyses) methodology. ${ }^{11}$ The search was undertaken in July 2017. We carried out electronic searches using MEDLINE (PubMed), SciELO, Web of Knowledge Cross Search (Thomson Scientific/ISI Web Services) and PsycINFO databases to select studies on coping involving caregivers of PwAD published in Portuguese, English or Spanish from January 2005 to July 2017.

The search terms were coping, caregivers, strategy, onset, adaptation, family, behavior, dementia and Alzheimer, in the following combinations: "coping AND caregivers AND Alzheimer," "coping AND strategy AND Alzheimer," "coping AND onset AND Alzheimer," coping AND strategy AND Alzheimer," "coping AND strategy AND early AND Alzheimer," "coping AND strategy AND early AND Alzheimer AND caregivers," "coping AND adaptation AND Alzheimer AND caregivers," "coping AND family AND Alzheimer," "coping AND onset AND family AND Alzheimer," "coping AND behavior AND family AND onset AND Alzheimer," "coping AND behavior AND family AND onset AND dementia," "coping AND onset AND dementia," "coping AND strategy AND behavior AND Alzheimer," "coping AND strategy AND onset AND Alzheimer," "coping AND adaptation AND behavior AND strategy AND onset AND Alzheimer," "coping AND strategy AND adaptation AND onset AND dementia."

The following inclusion criteria were taken into consideration: cross-sectional or longitudinal studies; randomized or non-randomized, controlled or not controlled studies on coping strategies of PWAD caregivers.

We excluded studies assessing caregivers of people with other pathologies and dementias; systematic reviews, meta-analyses or articles without a patient sample; studies on healthy aging; pharmacological 
studies; studies on the effectiveness of interventions that have not been designed to investigate coping; and validation of instruments.

The selected abstracts were read by two authors (AMFM and MCND); whenever the information present in the abstract was not enough to identify inclusion and exclusion criteria, the full text was retrieved. Afterwards, four authors (AMFM, NK, MATB and MCND) independently reviewed the full texts of the remaining papers and had consensus meetings to discuss any disagreement about inclusion. When necessary, a third coauthor (RLS) was involved to help clarify study eligibility. We categorized the included studies according to their design, samples, methods and results. We also categorized the coping strategies in emotion-focused, problem-focused, and dysfunctional.

\section{Results}

The database search yielded a total of 2,277 articles, of which 932 were identified in Thomson Scientific Web of Knowledge Cross Search, 1,113 in PubMed, 15 in SciELO and 217 in PsycINFO. Once 823 duplicates were removed, 1,388 articles were screened and analyzed according to the inclusion criteria. The reference lists were also scanned and 66 articles were manually selected. After application of inclusion and exclusion criteria, the total number was reduced to 24 articles. Figure 1 shows the article selection process.

\section{Definition of coping}

All the analyzed studies defined coping as the process by which the person tries to manage stress. ${ }^{5}$
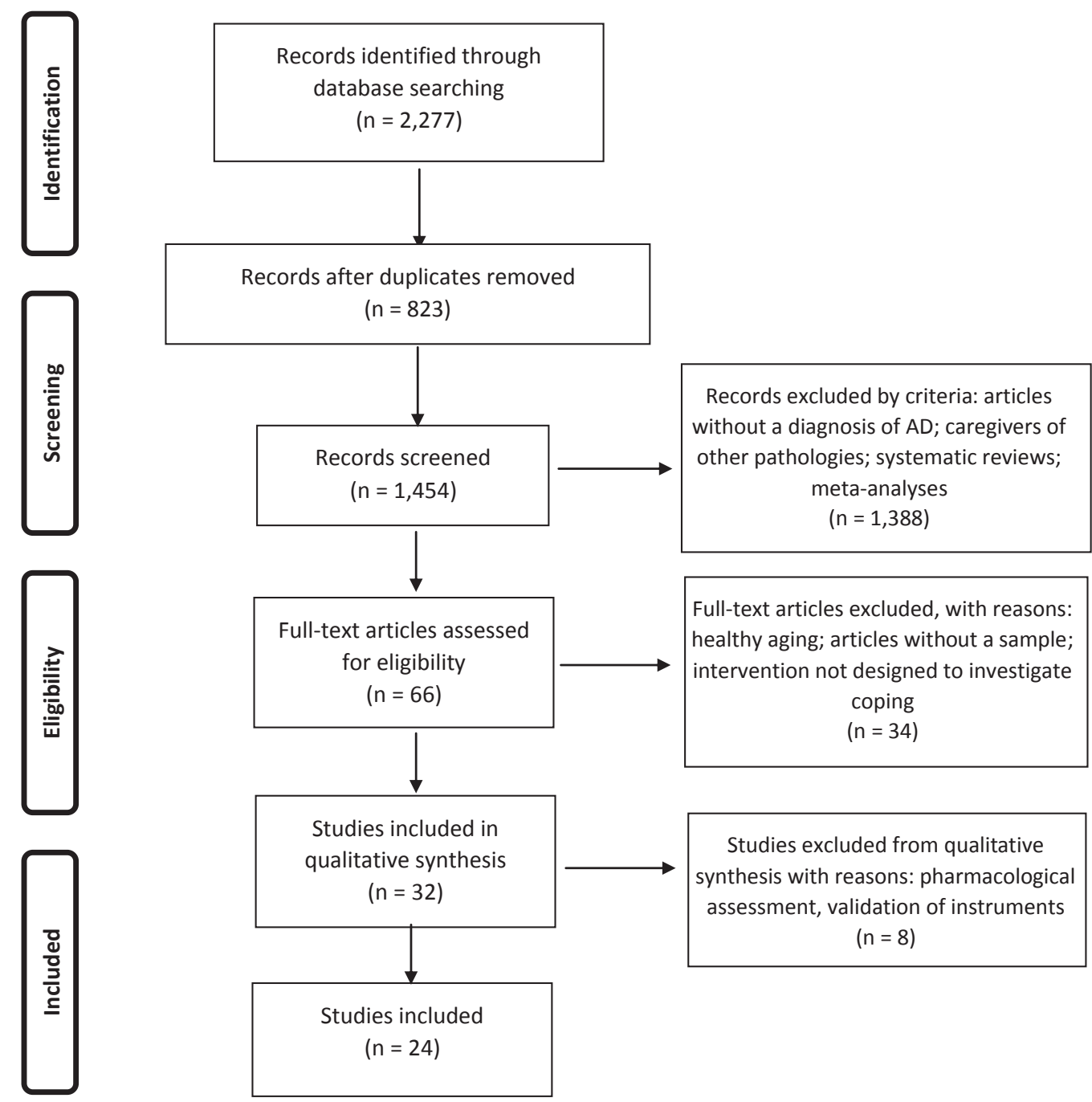

Figure 1 - Article selection process. AD = Alzheimer disease. 


\section{Design}

We found 19 studies with a cross-sectional design, ${ }^{4,12-29} 3$ randomized studies $7,30,31$ and 2 longitudinal studies. ${ }^{32,33}$

\section{Coping strategies}

Emotion-focused coping was the intervention strategy most frequently mentioned $(n=10) \cdot 1,7,15,17-20,22,25,30$ Seven studies mentioned problem-focused strategies $4,26-29,31,33$ and one ${ }^{13}$ mentioned only dysfunctional coping. Eight studies $4,14,16,21,23,24,32$ mentioned two or more strategies at the same time.

\section{Types of intervention}

\section{Emotion-focused coping strategies}

The findings showed reduction in caregiver anxiety, ${ }^{7,15,20,22,25,30,32}$ depression, $7,15,22,25,30$ behavioral and psychological symptoms (BPS), $7,12,14,24$ burnout 7,19 and stress, ${ }^{30}$ significant proportional effects on burden, ${ }^{15,20,32}$ greater improvement in average systolic and diastolic blood pressure ${ }^{30}$ and improvement in care outcomes of PwAD. ${ }^{16}$ Negative emotional coping strategies were associated with increased cumulative health risk and increased likelihood of weight gain. ${ }^{18}$

The most frequently used emotion-focused strategies were: acceptance (accepting the reality of the fact that it has happened/learning to live with it); emotional support (getting emotional support/comfort and understanding from others); humor (making jokes about it/making fun of the situation); positive reframing (trying to see it in a different light, make it seem more positive/look for something good in it); religion (trying to find comfort in my religious or spiritual beliefs/praying or meditating). ${ }^{23,32}$

\section{Problem-focused coping strategies}

These strategies were related to greater improvement of depressive symptoms, ${ }^{25,31}$ anxiety, ${ }^{20,25}$ perceived stress, ${ }^{4,28}$ burden, ${ }^{21}$ reduction of BPS $^{12,14,24}$ and reduction of $\mathrm{D}$-dimer levels. ${ }^{26}$ Also, the results showed that these strategies increased the sense of control, ${ }^{27}$ improved care outcomes of PWAD ${ }^{16}$ and slowed dementia progression. ${ }^{33}$ One study did not find a significant difference between usual care and the intervention in depressive symptoms in PWAD or their family caregivers. ${ }^{17}$

The problem-focused strategies most frequently used were: active coping (concentrating my efforts on doing something about the situation I'm in/taking action to try to make it better); instrumental support (getting help and advice from other people/trying to get advice or help from others about what to do); planning (trying to come up with a strategy about what to do/ thinking hard about what steps to take)..$^{23,32}$

\section{Dysfunctional coping strategies}

The findings showed that these strategies partially mediate the association between PWAD problem behaviors and depressive symptoms. ${ }^{32}$ Two studies reported that the avoidance-focused coping strategy increased caregiver burden levels. ${ }^{20,21}$

The most frequently used dysfunctional coping strategies were: behavioral disengagement (giving up trying to deal with it/the attempt to cope); denial (saying to myself this isn't real/refusing to believe that it has happened); self-distraction (turning to work or other activities to take my mind off things/doing something to think about it less); self-blame (criticizing myself/ blaming myself for things that happened); substance use (using alcohol or other drugs to make myself feel better/to help me get through it); venting (saying things to let my unpleasant feelings escape/expressing my negative feelings). . $3,32^{23}$

The coping intervention most frequently used was interview with caregivers. 4,13-16,18-24,28,29,32,33 Other interventions included: a manual-based coping strategy program ${ }^{25}$; photographs ${ }^{27}$; Strategies for RelaTives - START intervention7; videos adapted for Alzheimer disease or related dementias (ADRD); exercises and homework; and one telephone coaching call per week. ${ }^{30}$ Moreover, one study collected five saliva samples daily for two days ${ }^{17}$; and another used hypercoagulable D-dimer measurements at 3 time points. ${ }^{26}$

The categorization of the articles according the type of the coping strategies is shown in Table 1.

\section{Discussion}

We systematically reviewed coping strategies used by caregivers of PWAD. In general, the studies indicated the efficacy of coping strategies in ameliorating the effects of neuropsychiatric and clinical problems of PWAD on the caregivers' psychological and physical health. However, we observed a considerable heterogeneity of intervention techniques and methods of evaluation, which made it difficult to understand how to use the techniques in specific situations.

\section{Emotion-focused coping strategies}

Psychological distress and its consequences, such as anxiety7,12,15,19,23-25,30,32 and depression, 7,12,15,19,25,30,32 were mentioned in most studies. Religious coping strategy was combined with other emotional coping strategies or support for caregivers. ${ }^{4,16-18,22}$ The impact of the caregiving burden on the caregiver's health was described in two studies, ${ }^{20,21}$ showing that increased 
Table 1 - Description of the coping strategies

\begin{tabular}{|c|c|c|c|c|c|c|}
\hline Study & Sample & Design & Instruments & Objectives & Intervention & Results \\
\hline $\begin{array}{l}\text { Geiger et } \\
\text { al. }^{21}\end{array}$ & $\begin{array}{l}\mathrm{N}=138 \text { male } \\
\mathrm{AD} \text { caregivers }\end{array}$ & $\begin{array}{l}\text { Cross- } \\
\text { sectional }\end{array}$ & $\begin{array}{l}\text { CITS } \\
\text { ZBI } \\
\text { RMBPC }\end{array}$ & $\begin{array}{l}\text { Examine the effects of } \\
\text { coping strategies focused on } \\
\text { task, emotion and avoidance, } \\
\text { overload of male caregivers } \\
\text { of AD patients. }\end{array}$ & $\begin{array}{l}3 \text { coping strategies: } \\
\text { problem-focused, } \\
\text { emotion-focused, and } \\
\text { dysfunctional. }\end{array}$ & $\begin{array}{l}\text { Task focused on the highest reported } \\
\text { coping strategy. Emotion-focused } \\
\text { and avoidance-focused coping each } \\
\text { showed significant proportional } \\
\text { effects on burden. }\end{array}$ \\
\hline $\begin{array}{l}\text { Raggi, et } \\
\text { al. } 28\end{array}$ & $\begin{array}{l}\mathrm{N}=73 \mathrm{AD} \\
\text { primary } \\
\text { caregiver }\end{array}$ & $\begin{array}{l}\text { Cross- } \\
\text { sectional }\end{array}$ & $\begin{array}{l}\text { PWAD: CDR, GDS, } \\
\text { ADL, NPI, CIRS } \\
\text { Caregivers: CBI, } \\
\text { NPI }\end{array}$ & $\begin{array}{l}\text { Determine the severity of } \\
\text { caregiver burden in relation } \\
\text { to cognitive, behavioral, } \\
\text { functional, mood, mobility, } \\
\text { and comorbidity status of } \\
\text { patients; investigate coping } \\
\text { strategies for caregivers } \\
\text { according to their distress; } \\
\text { and assess psychosocial and } \\
\text { medical variables related to } \\
\text { caregiver burden. }\end{array}$ & $\begin{array}{l}\text { Problem-focused } \\
\text { coping strategies. }\end{array}$ & $\begin{array}{l}\text { The severity of caregiver distress } \\
\text { was correlated with specific coping } \\
\text { strategies, such as seeking for social } \\
\text { support, using avoidance behaviors } \\
\text { and focusing on problems. }\end{array}$ \\
\hline $\mathrm{Li}$, et al. ${ }^{7}$ & $\begin{array}{l}\mathrm{N}=260 \text { family } \\
\text { carers of PwD }\end{array}$ & Randomized & $\begin{array}{l}\text { START } \\
\text { HADS } \\
\text { BRCOPE }\end{array}$ & $\begin{array}{l}\text { Test the hypothesis that } \\
\text { increased emotion-focused } \\
\text { coping at } 4 \text { months would } \\
\text { mediate the effects of } \\
\text { the START intervention } \\
\text { in reducing psychological } \\
\text { morbidity symptoms at } 8 \\
\text { months; and test whether } \\
\text { this mechanism was } \\
\text { moderated by baseline } \\
\text { morbidity. }\end{array}$ & $\begin{array}{l}\text { Emotion-focused } \\
\text { coping strategies. } \\
\text { Eight-session START } \\
\text { intervention. The } \\
\text { intervention was based } \\
\text { on the stress appraisal } \\
\text { and coping model. } \\
\text { Treatment-as-usual. }\end{array}$ & $\begin{array}{l}\text { The strategies brought psychological } \\
\text { benefits to morbidity in different } \\
\text { mechanisms of action. The more } \\
\text { psychologically distressed caregivers } \\
\text { increased their emotion-focused } \\
\text { coping strategy and did not diminish } \\
\text { their dysfunctional coping, while } \\
\text { others improved burnout through } \\
\text { this mechanism. }\end{array}$ \\
\hline $\begin{array}{l}\text { Williams et } \\
\text { al. }{ }^{27}\end{array}$ & $\begin{array}{l}\mathrm{N}=13 \\
\text { caregivers of } \\
\text { people with } \\
\text { either stroke } \\
(\mathrm{n}=5) \text { or } \\
\text { dementia ( } \mathrm{n} \\
=8)\end{array}$ & $\begin{array}{l}\text { Cross- } \\
\text { sectional }\end{array}$ & $\begin{array}{l}\text { Semi-structured } \\
\text { interviews }\end{array}$ & $\begin{array}{l}\text { Explore coping strategies } \\
\text { that caregivers use to care } \\
\text { for the elderly. }\end{array}$ & $\begin{array}{l}\text { Problem-focused } \\
\text { coping strategies. } \\
\text { Use of photographs } \\
\text { that caregivers } \\
\text { took exemplifying } \\
\text { their caregiving } \\
\text { experiences to elicit } \\
\text { their description of how } \\
\text { they made sense of } \\
\text { caregiving. }\end{array}$ & $\begin{array}{l}\text { Caregivers adopted active and } \\
\text { information seeking techniques to } \\
\text { deal with current problems and } \\
\text { to increase their sense of control, } \\
\text { but avoidant techniques when } \\
\text { considering future logistics of } \\
\text { caregiving and when feeling helpless } \\
\text { due to the burden they faced. }\end{array}$ \\
\hline $\begin{array}{l}\text { Sun \& } \\
\text { Hodge }^{22}\end{array}$ & $\begin{array}{l}\mathrm{N}=209 \text { Latino } \\
\text { caregivers } \\
\text { included with } \\
\text { parents in USA }\end{array}$ & $\begin{array}{l}\text { Cross- } \\
\text { sectional }\end{array}$ & $\begin{array}{l}\text { REACH } \\
\text { MMSE } \\
\text { IADL } \\
\text { ADL } \\
\text { ZBI } \\
\text { CES-D }\end{array}$ & $\begin{array}{l}\text { Examine the effects of } \\
\text { spirituality and religion on } \\
\text { depression in a sample of } \\
\text { Latino AD caregivers. }\end{array}$ & $\begin{array}{l}\text { Emotion-focused } \\
\text { coping strategies. }\end{array}$ & $\begin{array}{l}\text { The findings indicate that church } \\
\text { attendance moderates the } \\
\text { relationship between subjective } \\
\text { forms of stress and depression in } \\
\text { tandem with exhibiting direct effects } \\
\text { on depression. }\end{array}$ \\
\hline $\begin{array}{l}\text { Tschanz et } \\
\text { al. }^{33}\end{array}$ & $\begin{array}{l}\mathrm{N}=226 \\
\text { persons with } \\
\text { dementia and } \\
\text { their caregivers } \\
\text { assessed semi- } \\
\text { annually for up } \\
\text { to } 6 \text { years }\end{array}$ & Longitudinal & $\begin{array}{l}\text { WCCL-R } \\
\text { MMSE } \\
\text { CDR }\end{array}$ & $\begin{array}{l}\text { Examine whether caregiver } \\
\text { coping strategies predict } \\
\text { dementia progression in a } \\
\text { population-based sample. }\end{array}$ & $\begin{array}{l}\text { Problem-focused } \\
\text { coping strategies. }\end{array}$ & $\begin{array}{l}\text { Caregiver coping strategies are } \\
\text { associated with slower dementia } \\
\text { progression. }\end{array}$ \\
\hline $\begin{array}{l}\text { Livingston et } \\
\text { al. }{ }^{25}\end{array}$ & $\begin{array}{l}\mathrm{N}=260 \text { carers } \\
\text { of family } \\
\text { members with } \\
\text { dementia }\end{array}$ & $\begin{array}{l}\text { Cross- } \\
\text { sectional }\end{array}$ & $\begin{array}{l}\text { HADS } \\
\text { QOL-AD } \\
\text { CTS2 } \\
\text { NPI } \\
\text { CDR } \\
\text { BRCOPE } \\
\text { START }\end{array}$ & $\begin{array}{l}\text { Assess whether a manual- } \\
\text { based coping strategy } \\
\text { compared with treatment } \\
\text { as usual reduces depression } \\
\text { and anxiety symptoms in } \\
\text { carers of family members } \\
\text { with dementia. }\end{array}$ & $\begin{array}{l}\text { Emotion-focused } \\
\text { coping strategies. A } \\
\text { manual-based coping } \\
\text { strategy program } \\
\text { can be delivered by } \\
\text { graduate psychologists } \\
\text { without clinical } \\
\text { training. }\end{array}$ & $\begin{array}{l}\text { Mean total scores on HADS were } \\
\text { lower in the intervention group than } \\
\text { in the treatment as usual group over } \\
\text { the 8-month evaluation period. }\end{array}$ \\
\hline Alma et al. ${ }^{14}$ & $\begin{array}{l}\mathrm{N}=11 \\
\text { caregivers }\end{array}$ & $\begin{array}{l}\text { Cross- } \\
\text { sectional }\end{array}$ & $\begin{array}{l}\text { Semi-structured } \\
\text { interviews }\end{array}$ & $\begin{array}{l}\text { Explore the possible } \\
\text { differences in social support } \\
\text { for coping mechanisms and } \\
\text { help-seeking behaviors in } \\
\text { Chinese caregivers. }\end{array}$ & $\begin{array}{l}\text { Emotion-focused } \\
\text { coping strategies } \\
\text { and problem-focused } \\
\text { coping strategies; } \\
\text { coping strategies } \\
\text { focused on self- } \\
\text { regulation, patient and } \\
\text { family obligations. }\end{array}$ & $\begin{array}{l}\text { Different standards of flexibility in } \\
\text { handling and seeking social support } \\
\text { to care. }\end{array}$ \\
\hline $\begin{array}{l}\text { García- } \\
\text { Alberca et } \\
\text { al. }^{24}\end{array}$ & $\begin{array}{l}\mathrm{N}=80 \text { patients } \\
\text { with } \mathrm{AD} \text { and } \\
\text { their primary } \\
\text { caregivers. }\end{array}$ & $\begin{array}{l}\text { Cross- } \\
\text { sectional }\end{array}$ & $\begin{array}{l}\text { Patients: MMSE, } \\
\text { B-ADL, GDTS, NPI } \\
\text { Caregivers: ZBI, } \\
\text { BDI, STAI, CSI }\end{array}$ & $\begin{array}{l}\text { Determine whether caregiver } \\
\text { coping strategies are } \\
\text { independently associated } \\
\text { with BPS in AD after } \\
\text { accounting for patient } \\
\text { characteristics. }\end{array}$ & $\begin{array}{l}\text { Two coping strategies: } \\
\text { task-focused, emotion- } \\
\text { focused }\end{array}$ & $\begin{array}{l}\text { Coping strategies are associated } \\
\text { with BPS regardless of patient } \\
\text { characteristics. Interventions to } \\
\text { reduce BPS should focus on which } \\
\text { psychological coping strategies } \\
\text { caregivers use. }\end{array}$ \\
\hline
\end{tabular}


Table 1 (cont.)

\begin{tabular}{|c|c|c|c|c|c|c|}
\hline Study & Sample & Design & Instruments & Objectives & Intervention & Results \\
\hline $\begin{array}{l}\text { Bruvik et } \\
\text { al. }^{31}\end{array}$ & $N=230$ & Randomized & $\begin{array}{l}\text { RSS } \\
\text { NPI } \\
\text { PSMS } \\
\text { IADL } \\
\text { MMSE } \\
\text { GDS }\end{array}$ & $\begin{array}{l}\text { Investigate the association } \\
\text { between coping, care of local } \\
\text { extent and burden of family } \\
\text { caregivers of PwAD. }\end{array}$ & $\begin{array}{l}\text { Problem-solving coping } \\
\text { strategies method in } \\
\text { steps: } 1 \text { ) definition } \\
\text { of a problem, as } \\
\text { concrete as possible; } \\
\text { 2) brainstorming, } \\
\text { all proposals to be } \\
\text { recorded; } 3 \text { ) discussing } \\
\text { the proposed solutions, } \\
\text { pros and cons; } 4 \text { ) } \\
\text { choosing a solution } \\
\text { or a combination of } \\
\text { solutions }\end{array}$ & $\begin{array}{l}\text { The trial did not show a significant } \\
\text { difference between usual care and } \\
\text { the intervention on depressive } \\
\text { symptoms in PwAD or their family } \\
\text { caregivers. }\end{array}$ \\
\hline $\begin{array}{l}\text { Merritt \& } \\
\text { McCallum }{ }^{17}\end{array}$ & $\begin{array}{l}\mathrm{N}=78 \\
30 \text { African- } \\
\text { American } \\
\text { female dementia } \\
\text { caregivers and } \\
48 \text { African- } \\
\text { American non- } \\
\text { caregivers }\end{array}$ & $\begin{array}{l}\text { Cross- } \\
\text { sectional }\end{array}$ & $\begin{array}{l}\text { RCOPE } \\
\text { IADL } \\
\text { RMBPC } \\
\text { SSD }\end{array}$ & $\begin{array}{l}\text { Examine the moderating role } \\
\text { of religious coping (positive, } \\
\text { negative, and combined) } \\
\text { in the connection of care } \\
\text { recipient functional status } \\
\text { with diurnal salivary cortisol } \\
\text { patterns among dementia } \\
\text { family caregivers. }\end{array}$ & $\begin{array}{l}\text { Emotion-focused } \\
\text { coping strategies. } \\
\text { Five saliva samples } \\
\text { were collected daily } \\
\text { (at awakening, 9am, } \\
12 \mathrm{pm}, 5 \mathrm{pm}, \text { and } 9 \mathrm{pm} \text { ) } \\
\text { for two straight days. }\end{array}$ & $\begin{array}{l}\text { The RCOPE by RMBPC interaction } \\
\text { was significant. Among caregivers } \\
\text { who showed higher RMBPC scores, } \\
\text { higher combined and positive (but } \\
\text { not negative) RCOPE scores were } \\
\text { unexpectedly associated with } \\
\text { increasingly flatter cortisol slopes. }\end{array}$ \\
\hline $\begin{array}{l}\text { García- } \\
\text { Alberca et } \\
\text { al. }{ }^{12}\end{array}$ & $\begin{array}{l}\mathrm{N}=80 \text { patients } \\
\text { and caregivers }\end{array}$ & $\begin{array}{l}\text { Cross- } \\
\text { sectional }\end{array}$ & $\begin{array}{l}\text { Patients: MMSE, } \\
\text { B-ADL, GDTS, NPI } \\
\text { Caregivers: ZBI, } \\
\text { BDI, STAI, CSI }\end{array}$ & $\begin{array}{l}\text { Investigate the relationship } \\
\text { between anxiety and } \\
\text { depression, and engagement } \\
\text { and disengagement coping } \\
\text { strategies, in caregivers of } \\
\text { PWAD in an epidemiological } \\
\text { population. }\end{array}$ & $\begin{array}{l}\text { Two coping strategies: } \\
\text { emotion-focused } \\
\text { coping strategies } \\
\text { and problem-focused } \\
\text { coping strategies. }\end{array}$ & $\begin{array}{l}\text { Coping strategies were associated } \\
\text { with BPS regardless of patient } \\
\text { characteristics. Interventions to } \\
\text { reduce BPS should focus on which } \\
\text { psychological coping strategies } \\
\text { caregivers use. }\end{array}$ \\
\hline Lim et al. ${ }^{16}$ & $\begin{array}{l}\mathrm{N}=107 \\
\text { Asian family } \\
\text { caregivers of } \\
\text { persons with } \\
\text { dementia in } \\
\text { Singapore }\end{array}$ & $\begin{array}{l}\text { Cross- } \\
\text { sectional }\end{array}$ & $\begin{array}{l}\text { Demographic } \\
\text { questionnaire } \\
\text { RMBPC } \\
\text { ZBI } \\
\text { BRCOPE } \\
\text { SBI-15R } \\
\text { DMSS } \\
\text { PAC }\end{array}$ & $\begin{array}{l}\text { Examine the factors } \\
\text { associated with negative } \\
\text { and positive adjustment } \\
\text { outcomes among Asian } \\
\text { family caregivers of persons } \\
\text { with dementia in Singapore. }\end{array}$ & $\begin{array}{l}\text { Emotion-focused } \\
\text { coping strategies } \\
\text { and problem-focused } \\
\text { coping strategies. } \\
\text { Coping styles, } \\
\text { management strategies } \\
\text { in dementia, religion } \\
\text { and spirituality, } \\
\text { adjustment problems. }\end{array}$ & $\begin{array}{l}\text { Religion and spirituality gained } \\
\text { indirectly through the use of } \\
\text { encouragement. Caregivers should } \\
\text { aim coping strategies to improve } \\
\text { care outcomes for patients. }\end{array}$ \\
\hline $\begin{array}{l}\text { Romero- } \\
\text { Moreno et } \\
\text { al. }{ }^{29}\end{array}$ & $\begin{array}{l}\mathrm{N}=166 \\
\text { caregivers }\end{array}$ & $\begin{array}{l}\text { Cross- } \\
\text { sectional }\end{array}$ & $\begin{array}{l}\text { CJCS-R } \\
\text { RMBPC } \\
\text { RRS } \\
\text { TMMS-24 } \\
\text { CES-D } \\
\text { POMS } \\
\text { STAEI }\end{array}$ & $\begin{array}{l}\text { Analyze the dimensional } \\
\text { structure of the culture and } \\
\text { the effects of motives for } \\
\text { caregiving on stressors, } \\
\text { caregiver resources and } \\
\text { outcome variables. }\end{array}$ & $\begin{array}{l}\text { Problem-focused } \\
\text { coping strategies. } \\
\text { Interviews with } \\
\text { caregivers. }\end{array}$ & $\begin{array}{l}\text { No differences between groups were } \\
\text { found in frequency of behavioral } \\
\text { problems. }\end{array}$ \\
\hline $\begin{array}{l}\text { Rabinowitz } \\
\text { et al. }{ }^{18}\end{array}$ & $\begin{array}{l}\mathrm{N}=256 \text { Latina } \\
\text { and Caucasian } \\
\text { female } \\
\text { caregivers of } \\
\text { elderly relatives } \\
\text { with dementia }\end{array}$ & $\begin{array}{l}\text { Cross- } \\
\text { sectional }\end{array}$ & $\begin{array}{l}\text { MMSE } \\
\text { ADL } \\
\text { IADL } \\
\text { REACH } \\
\text { RMBPC } \\
\text { BRCOPE }\end{array}$ & $\begin{array}{l}\text { Primary analyses examined } \\
\text { the relationship between } \\
\text { religious coping (both } \\
\text { positive and negative) } \\
\text { and an overall index of } \\
\text { cumulative health risk. } \\
\text { Secondary analyses were } \\
\text { conducted on individual } \\
\text { health behaviors subsumed } \\
\text { in the broader index. }\end{array}$ & $\begin{array}{l}\text { Emotion-focused } \\
\text { coping strategies. } \\
\text { Caregivers were asked } \\
\text { to answer questions } \\
\text { about religious coping } \\
\text { strategies, religion and } \\
\text { their own health. }\end{array}$ & $\begin{array}{l}\text { Findings revealed that negative } \\
\text { religious coping was significantly } \\
\text { associated with increased cumulative } \\
\text { health risk. Positive religious } \\
\text { coping was predictive of decreased } \\
\text { cumulative health risk among } \\
\text { Latina caregivers but not among } \\
\text { Caucasians. Negative religious } \\
\text { coping was significantly associated } \\
\text { with both an increased likelihood for } \\
\text { weight gain and increased dietary } \\
\text { restriction. Positive religious coping } \\
\text { was associated with decreased } \\
\text { likelihood for weight gain in Latinas. }\end{array}$ \\
\hline $\begin{array}{l}\text { Williams et } \\
\text { al. }{ }^{30}\end{array}$ & $\begin{array}{l}\mathrm{N}=116 \\
\text { caregivers } \\
59 \text { VCS } \\
57 \text { wait list } \\
\text { group }\end{array}$ & Randomized & $\begin{array}{l}\text { Demographic } \\
\text { questionnaire } \\
\text { PSS } \\
\text { STAI } \\
\text { STAXI } \\
\text { CES-D } \\
\text { CMHS } \\
\text { CGSE } \\
\text { PSQI } \\
\text { Biomarkers: BP, } \\
\text { HR, SC }\end{array}$ & $\begin{array}{l}\text { Determine whether VCS } \\
\text { training with telephone } \\
\text { coaching reduces } \\
\text { psychosocial and biological } \\
\text { markers of distress in } \\
\text { primary caregivers of a } \\
\text { relative with ADRD }\end{array}$ & $\begin{array}{l}\text { Emotion-focused } \\
\text { coping strategies. } \\
\text { Video adapted for } \\
\text { ADRD family care } \\
\text { contexts; exercises } \\
\text { and homework for } \\
\text { each module presented } \\
\text { in an accompanying } \\
\text { workbook; and one } \\
\text { telephone coaching call } \\
\text { per week for five weeks } \\
\text { on each week's two } \\
\text { modules. }\end{array}$ & $\begin{array}{l}\text { Compared to controls, participants } \\
\text { who received VCS training plus } \\
\text { telephone coaching showed } \\
\text { significantly greater improvements in } \\
\text { depressive symptoms, trait anxiety, } \\
\text { perceived stress, and average } \\
\text { systolic and diastolic blood pressure } \\
\text { that were maintained over the } \\
6 \text {-month follow-up period. }\end{array}$ \\
\hline $\begin{array}{l}\text { Tan \& } \\
\text { Schneider }^{19}\end{array}$ & $\begin{array}{l}\mathrm{N}=6 \text { young } \\
\text { adult-child } \\
\text { caregivers of } \\
\text { PWAD }\end{array}$ & $\begin{array}{l}\text { Cross- } \\
\text { sectional }\end{array}$ & BCHUAT & $\begin{array}{l}\text { Understand the experiences } \\
\text { of young adult-child } \\
\text { caregivers, with a key focus } \\
\text { on the use of humor as } \\
\text { a coping strategy in this } \\
\text { caregiving circumstance. }\end{array}$ & $\begin{array}{l}\text { Emotion-focused } \\
\text { coping strategies. } \\
\text { Interview with } \\
\text { caregivers. }\end{array}$ & $\begin{array}{l}\text { Use of humor as a coping strategy } \\
\text { prevented caregiver burnout and } \\
\text { optimized patient care. }\end{array}$ \\
\hline
\end{tabular}

Continued on next page 
Table 1 (cont.)

\begin{tabular}{|c|c|c|c|c|c|c|}
\hline Study & Sample & Design & Instruments & Objectives & Intervention & Results \\
\hline $\begin{array}{l}\text { Di Mattei et } \\
\text { al. }^{4}\end{array}$ & $\begin{array}{l}\mathrm{N}=112 \\
\text { primary } \\
\text { caregivers }\end{array}$ & $\begin{array}{l}\text { Cross- } \\
\text { sectional }\end{array}$ & CBI, COPE & $\begin{array}{l}\text { Investigate which } \\
\text { sociodemographic and clinical } \\
\text { variables are significantly } \\
\text { associated with higher levels } \\
\text { of distress in caregivers, and } \\
\text { the relationship between } \\
\text { caregiver levels of distress } \\
\text { and the coping strategies they } \\
\text { adopt. }\end{array}$ & $\begin{array}{l}\text { Problem-focused } \\
\text { coping strategies. } \\
\text { Social support, } \\
\text { avoidance coping, } \\
\text { positive attitude, focus } \\
\text { on problem, religion }\end{array}$ & $\begin{array}{l}\text { Caregivers with the highest levels } \\
\text { of distress were characterized by } \\
\text { an impaired physical health status. } \\
\text { Avoidance coping may represent } \\
\text { a risk factor associated with } \\
\text { higher levels of distress. Active } \\
\text { and problem-focused approach to } \\
\text { stressful situations may act as a } \\
\text { protective factor. }\end{array}$ \\
\hline $\begin{array}{l}\text { Cooper et } \\
\text { al. }^{32}\end{array}$ & $\begin{array}{l}\mathrm{N}=93 \text { patients } \\
\text { and caregivers }\end{array}$ & Longitudinal & $\begin{array}{l}\text { Patients: HADS, } \\
\text { ZBI, BRCOPE, } \\
\text { SRRS, HSQ-12, } \\
\text { QOL-AD } \\
\text { Caregivers: MMSE, } \\
\text { ADAS-Cog, ADCS- } \\
\text { ADL, NPI }\end{array}$ & $\begin{array}{l}\text { Investigate the impact of } \\
\text { coping on the psychological } \\
\text { morbidity of caregivers of } \\
\text { people with dementia. }\end{array}$ & $\begin{array}{l}\text { Two coping strategies: } \\
\text { problem-focused, } \\
\text { emotion-focused. }\end{array}$ & $\begin{array}{l}\text { Using emotion-focused coping } \\
\text { strategies in response to caregiver } \\
\text { burden seemed to protect caregivers } \\
\text { from developing higher anxiety } \\
\text { levels a year later; however, using } \\
\text { problem-focused strategies did not. }\end{array}$ \\
\hline Chun et al. ${ }^{15}$ & $\begin{array}{l}\mathrm{N}=171 \\
\text { caregivers } \\
64 \text { Koreans } \\
\text { residing in } \\
\text { Korea, } 53 \\
\text { Korean- } \\
\text { Americans } \\
\text { and } 54 \text { White- } \\
\text { Americans living } \\
\text { in the USA }\end{array}$ & $\begin{array}{l}\text { Cross- } \\
\text { sectional }\end{array}$ & $\begin{array}{l}\text { Sociodemographic } \\
\text { questionnaire } \\
\text { RMBPC } \\
\text { STAI } \\
\text { CES-D } \\
\text { ZBI }\end{array}$ & $\begin{array}{l}\text { Compare models of } \\
\text { emotional distress on three } \\
\text { groups of caregivers of } \\
\text { patients with dementia: } \\
\text { Korean residents in Korea, } \\
\text { White-Americans and } \\
\text { Korean-Americans residing in } \\
\text { the USA. }\end{array}$ & $\begin{array}{l}\text { Emotion-focused } \\
\text { coping strategies. } \\
\text { Caregivers were } \\
\text { interviewed } \\
\text { using structured } \\
\text { questionnaires. }\end{array}$ & $\begin{array}{l}\text { Patients with disruptive behavior } \\
\text { lead to caregiver burden and cause } \\
\text { anxiety and depression. Emotional } \\
\text { support is important for Korean- } \\
\text { Americans. }\end{array}$ \\
\hline $\begin{array}{l}\text { Riedijk et } \\
\text { al. }\end{array}$ & $\begin{array}{l}29 \text { FTD } \\
90 \text { AD } \\
34 \text { caregivers of } \\
\text { institutionalized } \\
\text { FTD }\end{array}$ & $\begin{array}{l}\text { Cross- } \\
\text { sectional }\end{array}$ & $\begin{array}{l}\text { MAASBED } \\
\text { NPI } \\
\text { HQOL } \\
\text { UCL }\end{array}$ & $\begin{array}{l}\text { Compare private caregivers } \\
\text { and caregivers of } \\
\text { institutionalized elderly and } \\
\text { to differentiate FTD caregiver } \\
\text { burden from AD caregiver } \\
\text { burden. }\end{array}$ & $\begin{array}{l}\text { Emotion-focused } \\
\text { coping strategies. }\end{array}$ & $\begin{array}{l}\text { Caregivers of FTD patients } \\
\text { institutionalized after shorter } \\
\text { dementia duration were most } \\
\text { burdened and affected in their HQoL. } \\
\text { Overall, passive coping strategies } \\
\text { were associated with increased } \\
\text { burden and decreased HQoL. }\end{array}$ \\
\hline $\begin{array}{l}\text { Cooper et } \\
\text { al. }^{23}\end{array}$ & $\begin{array}{l}\mathrm{N}=126 \text { family } \\
\text { caregivers }\end{array}$ & $\begin{array}{l}\text { Cross- } \\
\text { sectional }\end{array}$ & $\begin{array}{l}\text { Sociodemographic } \\
\text { questionnaire } \\
\text { HADS } \\
\text { BRCOPE }\end{array}$ & $\begin{array}{l}\text { Evaluate anxiety in } \\
\text { caregivers of PwAD and the } \\
\text { relationship with coping } \\
\text { strategies. }\end{array}$ & $\begin{array}{l}\text { Three coping } \\
\text { strategies: problem- } \\
\text { focused, emotion- } \\
\text { focused, and } \\
\text { dysfunctional-focused. }\end{array}$ & $\begin{array}{l}\text { Anxiety is more likely in caregivers } \\
\text { who do not use coping strategies. }\end{array}$ \\
\hline $\begin{array}{l}\text { Aschbacher } \\
\text { et al. }{ }^{26}\end{array}$ & $\begin{array}{l}60 \text { caregivers } \\
\text { and } 33 \text { non- } \\
\text { caregivers } \\
\text { control group }\end{array}$ & $\begin{array}{l}\text { Cross- } \\
\text { sectional }\end{array}$ & $\begin{array}{l}\text { WAYS-R } \\
\text { SP } \\
\text { HAM-D } \\
\text { HARS } \\
\text { PPS } \\
\text { WOC }\end{array}$ & $\begin{array}{l}\text { Assess } 1 \text { ) whether } \\
\text { coping processes affect } \\
\text { hemostatic reactivity to } \\
\text { acute psychological stress, } \\
\text { and } 2 \text { ) if these effects } \\
\text { differ substantially between } \\
\text { caregivers and non- } \\
\text { caregivers. }\end{array}$ & $\begin{array}{l}\text { Problem-focused } \\
\text { coping strategies. } \\
\text { The marker } \\
\text { hypercoagulable } \\
\text { D-dimer was measured } \\
\text { at three time points: } \\
\text { baseline, post-speech } \\
\text { immediately, and } \\
\text { during recovery (15 } \\
\text { minutes post-speech). }\end{array}$ & $\begin{array}{l}\text { Reduction of D-dimer levels at all } \\
\text { points. Caregivers with low levels } \\
\text { of PPS showed greater increase in } \\
\text { baseline D-dimer than the control } \\
\text { group. }\end{array}$ \\
\hline $\begin{array}{l}\text { Mausbach, } \\
\text { et al. }{ }^{13}\end{array}$ & $\begin{array}{l}\mathrm{N}=95 \text { spousal } \\
\text { caregivers }\end{array}$ & $\begin{array}{l}\text { Cross- } \\
\text { sectional }\end{array}$ & $\begin{array}{l}\text { Demographic } \\
\text { questionnaire } \\
\text { CDR } \\
\text { IMED } \\
\text { HDRS } \\
\text { BDI } \\
\text { WAYS-R } \\
\text { BSI }\end{array}$ & $\begin{array}{l}\text { Test a mediational model of } \\
\text { the associations between } \\
\text { patient problem behaviors, } \\
\text { escape-avoidance coping, } \\
\text { and depressive symptoms in } \\
\text { AD caregivers. }\end{array}$ & $\begin{array}{l}\text { Dysfunctional coping } \\
\text { strategies. Caregivers } \\
\text { were asked to answer } \\
\text { questions about } \\
\text { themselves and about } \\
\text { patients. }\end{array}$ & $\begin{array}{l}\text { Escape-avoidance coping partially } \\
\text { mediates the association between } \\
\text { patient problem behaviors and } \\
\text { depressive symptoms among elderly } \\
\text { caregivers of spouses with dementia. }\end{array}$ \\
\hline
\end{tabular}

$A D=$ Alzheimer disease; ADAS-Cog = Alzheimer's Disease Assessment Scale - Cognition; ADCS-ADL = Alzheimer's Disease Cooperative Study Inventory Activities of Daily Living; $A D L=$ activities of daily living; $A D R D=$ Alzheimer disease or related dementia; $B-A D L=B a y e r$ Activities of Daily Living Scale; $B C H U A T$ = Buffum Caregiver Humor use Assessment Tool; BDI = Beck Depression Inventory; BP = blood pressure; BPS = behavioral and psychological symptoms; BRCOPE = Brief Religious Coping Scale; BSI = Brief Symptom Inventory; CBI = Caregiver Burden Inventory; CDR = Clinical Dementia Rating; CES-D = Center for Epidemiologic Studies Depression Scale; CGSE = Revised Scale for Caregiving Self-Efficacy; CIRS = Cumulative Illness Rate Scale; CITS = Coping Inventory for Task Stressors; CJCS-R = Cultural Justifications for Caregiving Scale - Revised; CMHS = Cook-Medley Hostility Scale; COPE = Coping Orientation to Problem Experienced; CSI = Coping Strategies Inventory; CTS2 = Revised Conflict Tactics Scales; DMSS = Dementia Management Strategies Scale; FTD = frontotemporal dementia; GDS = Geriatric Depression Scale; GDTS = Global Deterioration Scale; HADS = Hospital Anxiety and Depression Scale; HAM-D = Hamilton Rating Scale for Depression; HARS = Hamilton Anxiety Rating Scale; HDRS = Hamilton Depression Rating Scale; HQoL = health-related quality of life; $\mathrm{HR}=$ heart rate; HSQ-12 = Health Status Questionnaire; IADL = instrumental activities of daily living; IMED = interim medical history questionnaire; MAASBED $=$ Maastricht Study of Behavior in Dementia; MMSE = Mini Mental State Exam; NPI = Neuropsychiatric Inventory; PAC = Positive Aspects of Caregiving; POMS = Profile of Mood States; PPS = planful problem solving; PSMS = Physical Self-Maintenance Scale; PSQI = Pittsburgh Sleep Quality Index; PSS = Perceived Stress Scale; PwAD = People with Alzheimer disease; PwD = People with dementia; QOL-AD = Quality of Life in Alzheimer's Disease; RCOPE = Religious Coping Scale; REACH = Resource for Enhancing Alzheimer's Caregivers Health; RMBPC = Revised Memory and Behavior Checklist; RRS = Ruminative Responses Scale; RSS = Relative Stress Scale; SBI-15R = Systems of Belief Inventory-15R; SC = salivary cortisol; SP = stress protocol; SRRS = Social Readjustment Rating Scale; SSD = saliva samples daily; STAEI = State-Trait Anger Expression Inventory; STAI = State-Trait Anxiety Inventory; START = Strategies for RelaTives; STAXI = State-Trait Anger Inventory; TMMS-24 = Trait Meta-Mood Scale; UCL = Utrecht Coping List; VCS = video-based coping skills; WAYS-R = Revised Ways of Coping; WCCL-R = Ways of Coping Checklist-Revised; WOC = Ways of Coping; ZBI = Zarit Burden Inventory. 
usage of avoidance-focused coping increases caregiver burden levels.

In Cooper et al., ${ }^{32}$ caregivers used all three types of coping strategies. They found a difference in the efficacy of specific types of coping strategies. The use of all three strategies did not protect against the impact of greater burden or anxiety. However, those who responded by using more emotion-focused strategies were less anxious one year later.

Alma et al. ${ }^{14}$ studied coping and help-seeking behavior among Hong Kong Chinese family caregivers of older people diagnosed with AD. The study found evidence of distinct coping strategies that focused upon internal self-regulation, forbearance and family obligations. In terms of help-seeking behavior, most caregivers expressed great concern about bothering their family members. When there was a desperate need for help, they turned to community services. Results are discussed in the context of both traditional Chinese cultural values as well as the modern transformations of the Chinese society. In particular, Eastern philosophical teachings tend to focus on changing personal inner perception and thoughts rather than attempting to change the environment. ${ }^{14}$ Despite these limitations, the study has implications for rethinking aspects of the care for people with dementia and also for older people in general in both Hong Kong and other Chinese societies.

Chun et al. ${ }^{15}$ compared path models of emotional distress among three groups of caregivers of elderly patients with dementia: 64 Koreans residing in Korea, 53 Korean-Americans and 54 White-Americans living in the United States of America. The results support a common core model throughout the three groups: PWAD disruptive behavior leads to burden, which then increases caregiver depression and anxiety. Instrumental support was found to be an important factor for Korean caregivers, and emotional support was important for Korean-Americans. Only Korean-American caregivers appraised the disruptive behaviors, memory problems, and depression as burdensome. These findings suggest that stress and coping processes in caregivers from different cultures involve differences in the effects of PWAD problems and social support available to caregivers. Nevertheless, since that study did not have a non-caregiver comparison group, there is a possibility that the results were due to general ethnic or cross-national differences rather than to ethnicity or caregiving interactions.

García-Alberca et al. ${ }^{24}$ conducted a study to obtain new data regarding the association of emotion-focused and problem-solving coping strategies with psychological distress in AD caregivers. Eighty PWAD and their primary caregivers living in the community were recruited from local health services. Most caregivers reported higher anxiety and depression levels. Use of disengagement coping strategies and higher caregiver burden predicted anxiety and depression in the logistic regression. Caregivers were asked about coping strategies using a general measure of coping, and not inquiring about specific situations. Also, almost all measures in the study were self-reported. The extent to which the answers of the participants on coping strategy measures relate to actual behavior is debatable.

Li et al., ${ }^{7}$ in a randomized controlled trial, studied the relationship between anxiety and depression in family carers of PWAD. The decreased depression in the intervention group was mediated by increased emotion-focused coping only among carers with higher baseline depression scores. The treatment benefited family carers both in preventing and treating psychological morbidity, through different mechanisms of action. The most psychologically distressed carers increased their emotion-focused coping and did not decrease their dysfunctional coping, while others benefited, but not through this mechanism. The study did not measure plausible psychosocial treatment mechanisms other than coping.

Lim et al., ${ }^{16}$ in a cross-sectional study, used the stress and coping paradigm to examine the factors associated with negative and positive adjustment outcomes among Asian family caregivers of PWAD in Singapore. One hundred seven family caregivers completed measures assessing PwAD illness characteristics, general coping styles of caregivers, specific dementia management strategies, religion and spirituality, and caregiver adjustment outcomes of burden and gain. Multiple regressions revealed that behavioral problems in PWAD, dementia severity, and the use of behavioral disengagement and criticism as coping strategies were significant predictors of burden, accounting for $48 \%$ of the explained variance. The only significant predictor of gain was the use of encouragement as a specific dementia management strategy, explaining $18 \%$ of variance. Religion and spirituality predicted gain indirectly through the use of encouragement. The study had an exploratory nature and multiple comparisons were made without adjusting or decreasing significance levels, which increases the risk of type I error.

Livingston et al.25 assessed whether a manualbased coping strategy compared with treatment as usual reduced depressive and anxious symptoms in caregivers. A manual-based coping intervention comprising eight sessions and delivered by supervised psychology students was applied in 260 carers. The 
sample was divided as follows: 173 were randomized to the intervention and 87 to treatment as usual. Mean total scores on the Hospital Anxiety and Depression Scale were lower in the intervention group than in the treatment as usual group over the eight months' evaluation period. Carers in the intervention group were less likely to show depression and there was a trend towards reduced anxiety. The quality of life of carers was higher in the intervention group, but not for the recipient of care. In addition, carers in the intervention group reported less abusive behavior towards the recipient of care compared with those in the treatment as usual, although this result was not significant. The manual-based coping strategy may be considered effective in reducing affective symptoms and depression of caregivers.

Merrit et al. ${ }^{17}$ examined the moderating role of religious coping (positive, negative, and combined) in the connection of care recipient functional status with diurnal salivary cortisol patterns among $A D$ family caregivers. Thirty African-American female dementia caregivers and 48 African-American non-caregivers completed the Religious Coping Scale (RCOPE), Activities of Daily Living Scale (ADL) and Revised Memory and Behavior Problem Checklist (RMBPC) and collected five saliva samples daily for two straight days. Hierarchical regression tests with mean diurnal cortisol slope as the outcome illustrated, surprisingly, that higher combined and positive (but not negative) RCOPE scores were associated with increasingly flatter or worse cortisol slope scores for caregivers (but not for non-caregivers). Among caregivers who reported higher RMBPC scores, higher combined and positive (but not negative) RCOPE scores were also unexpectedly associated with increasingly flatter cortisol slopes. These results showed that being African-American, being a caregiver, and having higher positive religious coping may predict increased daily stress responses, mainly for those with higher PWAD behavioral problems. Since religious coping is a central coping strategy for African-American caregivers, it is vital that epidemiological assessments of religious coping in health and aging as well as tailored interventions focus on the unique reasons for this disparity.

Tan \& Schneider ${ }^{19}$ studied the use of humor of the young adult-child caregiver population as coping strategy. This research found that the experience of the young adult-child caregiver differs from that of other caregivers in the ability of using humor to relieve stress and provide an uplifting, inspirational feeling. Humor may be incorporated as a coping strategy for caregivers in the hope of preventing caregiver burnout and optimizing PWAD care.

\section{Problem-focused coping strategies}

Problem-focused coping strategies mostly used active coping interventions, in which caregivers concentrate the efforts on 1 ) doing something about the problem-situation, 4,16,26,27,33 and 2) planning, in which the caregiver tries to come up with a strategy about what to do, or thinks hard about what steps to take. $12,14,26,28,30$

Tschanz et al. ${ }^{33}$ suggested that the regular use of caregiver-focused coping was associated with a significant slower decline in cognition and function, a finding that considered the potential influence of environmental factors on the progression of dementia. However, the study did not show how caregivers perceived or appraised problems associated with caregiving, a potentially important factor that may influence both caregiver and patient outcomes.

Riedjik et al. ${ }^{25}$ studied informal caregivers of $A D$ and frontotemporal dementia in people living at home $(\mathrm{FTDH})$. FTDH and AD caregivers were compared regarding to caregiver burden, health-related quality of life (HQLL), and coping. They found that FTDH caregivers had more difficulties in finding support in the health care system, since FTDH is much less prevalent and known than AD. FTDH and AD caregivers did not differ significantly in their use of coping strategies. Both FTDH and AD caregivers made most use of active coping strategies and reassuring thoughts, and both groups made least use of passive coping strategies.

Aschbacher et al. ${ }^{26}$ longitudinally investigated 60 elderly community-dwelling spousal caregivers of PWAD and 33 non-caregiving controls to evaluate whether coping processes affected hemostatic reactivity to acute psychological stress, and whether these effects differed substantially between caregivers and non-caregivers. Participants were administered an acute stress test that required them to deliver a 3-minute speech challenge to the interviewer on an assigned topic. The hypercoagulability marker D-dimer was measured at three time points: baseline, immediately post-speech, and during recovery (15 minutes post-speech). They found that participants who endorsed greater levels of approach coping had decreased levels of D-dimer at all time points. A significant three-way interaction between problem solving, caregiver status and the temporal pattern of D-dimer was found, indicating that caregivers with low levels of problem solving exhibited greater increase in D-dimer from baseline to speech and recovery time points relative to controls. It may be possible that approach and problem-solving coping processes buffer the impact of acute psychological stressors on procoagulant activity. 
Williams et al. ${ }^{30}$ tried to determine whether videobased coping skills (VCS) training with telephone coaching reduced psychosocial and biological markers of distress in primary caregivers of a relative with ADRD. The results showed that, compared to controls, participants who received VCS training associated with telephone coaching had significantly greater improvement in depressive symptoms, anxiety, perceived stress, and average systolic and diastolic blood pressure, which were maintained over the six-month follow-up period. The study excluded ADRD caregivers who were unable to travel to participate in the training, although these individuals may have been among the most overburdened members of the population. Even though this research has shown that the more heavily burdened caregivers benefit most from interventions by excluding caregivers for logistical reasons, it may have underestimated the potential benefits of interventions. A second issue is that interventions often focused on the benefits of counseling and support groups that are unlikely to be available to caregivers or would be too costly.

\section{Dysfunctional coping strategies}

The analysis of dysfunctional coping strategies showed that they are not recommended or beneficial to approach caregiver problems. Dysfunctional strategies such as confrontation, escape and avoidance are considered negative for both the caregiver and the PWAD, and may have harmful consequences for the PwAD treatment.

The most used strategy was behavioral disengagement, in which the maladaptive strategy suggested a possible link between avoidant coping and depression and anxiety symptoms in caregivers. The avoidance-focused coping strategy involves purposely avoiding activities related to a stressor. ${ }^{29}$ Avoidance-focused coping includes strategies such as substance use or denial. Avoidance-focused coping is associated with significantly higher burden, decreased life satisfaction, and negative physical health outcomes compared to task- and emotion-focused coping. ${ }^{13}$

The results presented by García-Alberca et al. ${ }^{12}$ suggested that dysfunctional coping strategies are associated with caregiver anxiety and depression irrespective of demographic or clinical characteristics of either the PWAD or the caregiver, suggesting that coping is the most important predictor of caregiver psychological distress. This was a cross-sectional study and therefore the relationship between BPS and caregiver coping strategies may be bidirectional; the direction of causality should be reinforced by $a$ longitudinal study.

Mausbach et al. ${ }^{13}$ tested a mediational model of the associations between PwAD problem behaviors, escape-avoidance coping, and depressive symptoms in caregivers. Ninety-five spousal caregivers completed measures assessing their loved ones' frequency of problem behaviors, escape-avoidance coping, and depressive symptoms. Escape-avoidance coping partially mediated the association between PWAD problem behaviors and depressive symptoms among elderly caregivers of spouses with dementia.

Cooper et al. ${ }^{23}$ evaluated anxiety in caregivers of PWAD and its relationship with coping strategies. The findings showed that dysfunctional coping strategies and depression appeared to be the most important factors predicting caregiver anxiety. Anxious caregivers may be more likely to report their strategies negatively; these results do not clarify or affirm a direction of causality.

\section{Limitations}

In this systematic review, we included studies that were not randomized and controlled, which did not allow for an evaluation of exclusively gold standard interventions. In addition, we selected studies that did not describe the coping strategies used, which made it difficult to understand how the techniques were used in specific situations.

\section{Conclusions}

The development of coping strategies is fundamental for the management of caregiver psychological distress. The studies indicated the efficacy of coping strategies in the management of neuropsychiatric problems and clinical problems of PWAD, and also for the psychological and physical health of caregivers. We considered that dysfunctional coping strategies may have harmful consequences for the management of PWAD. Problemsolving strategies are effective when dealing with specific problems. Emotion-focused coping was the intervention strategy most frequently mentioned, and it may ameliorate depressive symptoms, anxiety, quality of life and caregiver burden. However, we observed a considerable heterogeneity of intervention techniques and methods of evaluation, which made it difficult to understand how to use the techniques in specific situations. There is a great need for more longitudinal studies that clearly describe the type of coping strategy used in relation to the studied outcomes.

Choosing the right coping strategy can bring benefits to PWAD and caregivers, and avoid stress and burden. Current findings suggest that caregivers may benefit PWAD in biopsychosocial aspects if they use the appropriate coping strategy. 


\section{Disclosure}

No conflicts of interest declared concerning the publication of this article.

\section{References}

1. American Psychiatry Association. Diagnostic and Statistical Manual of Mental Disorders, Fifth Edition (DSM-5). Arlington: American Psychiatric Association, 2013.

2. Contador I, Fernández-Calvo B, Palenzuela DL, Miguéis S, Ramos F. Prediction of burden in family caregivers of patients with dementia: a perspective of optimism based on generalized expectancies of control. Aging Ment Health. 2012;16:675-82.

3. Wilks SE, Croom B. Perceived stress and resilience in Alzheimer's disease caregivers: testing moderation and mediation models of social support. Aging Ment Health. 2008;12:357-65.

4. Di Mattei VE, Prunas A, Novella L, Marcone A, Cappa SF, Sarno L. The burden of distress in caregivers of elderly demented patients and its relationship with coping strategies. Neurol Sci. 2008;29:383-9.

5. Lazarus RS, Folkman S. Stress, appraisal, and coping. New York: Springer; 1984.

6. Zaumseil M, Schwarz S. Understandings of coping: A critical review of coping theories for disaster contexts. In: Zaumseil M, Schwarz S, von Vacano M. Cultural psychology of coping with disasters: the case of an earthquake in Java, Indonesia. New York: Springer-Verlag; 2014. p. 45-54.

7. Li R, Cooper C, Barber J, Rapaport P, Griffin M, Livingston G. Coping strategies as mediators of the effect of the START (strategies for RelaTives) intervention on psychological morbidity for family carers of people with dementia in a randomised controlled trial. J Affect Disord. 2014;168:298-305.

8. Goode KT, Haley WE, Roth DL, Ford GR. Predicting longitudinal changes in caregiver physical and mental health: a stress process model. J Health Psychol. 1998;17:190-8

9. Vitaliano PP, Russo J, Young HM, Teri L. Predictors of burden in spouse caregivers of individuals with Alzheimer's disease. Psychol Aging. 1991;6:392-402.

10. Vedhara K, Shanks N, Wilcock G, Lightman SL. Correlates and predictors of self-reported psychological and physical morbidity in chronic caregiver stress. J Health Psychol. 2001;6:101-19.

11. Moher D, Liberati A, Tetzlaff J, Altman DG; PRISMA Group. Preferred reporting items for systematic reviews and meta-analyses: the PRISMA statement. Ann Intern Med. 2009;151:264-9.

12. García-Alberca JM, Cruz B, Lara JP, Garrido V, Lara A, Gris E, et al. The experience of caregiving: the influence of coping strategies on behavioral and psychological symptoms in patients with Alzheimer's disease. Aging Ment Health. 2013;17:615-22.

13. Mausbach BT, Aschbacher $\mathrm{K}$, Patterson TL, Ancoli-Israel $\mathrm{S}$, Känel RV, Mills PJ, et al. Avoidant coping partially mediates the relationship between patient problem behaviors and depressive symptoms in spousal Alzheimer caregivers. Am J Geriatr Psychiatry. 2006;14:299-306.

14. Alma AU, Shardlow SM, Teng Y, Tsien T, Chan C. Coping strategies and social support-seeking behavior among Chinese caring for older people with dementia. Aging Soc. 2013;33:1422-41.

15. Chun M, Knight BG, Youn G. Differences in stress and coping models of emotional distress among Korean, Korean-American and White-American caregivers. Aging Ment Health. 2007;11:20-9.

16. Lim J, Griva K, Goh J, Hui Chionh L, Yap P. Coping strategies influence caregiver outcomes among Asian family caregivers of persons with dementia in Singapore. Alzheimer Dis Assoc Disord. 2011:25:34-41.

17. Merritt MM, McCallum TJ. Too much of a good thing? Positive religious coping predicts worse diurnal salivary cortisol patterns for overwhelmed African-American female dementia family caregivers. Am J Geriatr Psychiatry. 2013;21:46-56.

18. Rabinowitz YG, Hartlaub MG, Saenz EC, Thompson LW, GallagherThompson D. Is religious coping associated with cumulative health risk? An examination of religious coping styles and health behavior patterns in Alzheimer's dementia caregivers. J Relig Health. 2010;49:498-512.

19. Tan T, Schneider MA. Humor as a coping strategy for adult-child caregivers of individuals with Alzheimer's disease. Geriatr Nur. 2009;30:397-408.

20. Riedijk SR, De Vugt ME, Duivenvoorden HJ, Niermeijer MF, Van Swieten JC, Verhey FRJ, et al. Caregiver burden, health-related quality of life and coping in dementia caregivers: a comparison of frontotemporal dementia and Alzheimer's disease. Dement Geriatr Cogn Disord. 2006;22:405-12.

21. Geiger JR, Wilks SE, Lovelace LL, Chen Z, Spivey CA. Burden among male Alzheimer's caregivers: effects of distinct coping strategies. Am J Alzheimers Dis Other Demen. 2015;30:238-46.

22. Sun $F$, Hodge DR. Latino Alzheimer's disease caregivers and depression: using the stress coping model to examine the effects of spirituality and religion. J Appl Gerontol. 2014;33:291-315.

23. Cooper C, Katona C, Orrell M, Livingston G. Coping strategies and anxiety in caregivers of people with Alzheimer's disease: the LASER-AD study. J Affect Disord. 2006;90:15-20.

24. García-Alberca JM, Cruz B, Lara JP, Garrido V, Lara A, Gris E. Anxiety and depression are associated with coping strategies in caregivers of Alzheimer's disease patients: results from the MÁLAGA-AD study. Int Psychogeriatr. 2012;24:1325-34.

25. Livingston G, Barber J, Rapaport P, Knap M, Griffin M, King D, et al. Clinical effectiveness of a manual based coping strategy programme (START, STrAtegies for RelaTives) in promoting the mental health of carers of family members with dementia: pragmatic randomised controlled trial. BMJ. 2013;347:f6276.

26. Aschbacher $K$, Patterson $T L$, von Känel $R$, Dimsdale JE, Mills $\mathrm{PJ}$, Adler KA, et al. Coping processes and hemostatic reactivity to acute stress in dementia caregivers. Psychosom Med. 2005;67:964-71.

27. Williams KL, Morrison V, Robinson CA. Exploring caregiving experiences: caregiver coping and making sense of illness, Aging Ment Health. 2014;18:600-9.

28. Raggi A, Tasca D, Panerai S, Neri W, Ferri R. The burden of distress and related coping processes in family caregivers of patients with Alzheimer's disease living in the community. J Neurol Sci. 2015;358:77-81.

29. Romero-Moreno R, Márquez-González M, Losada A, López J. Motives for caring: relationship to stress and coping dimensions. Int Psychogeriatr. 2011;23:573-82.

30. Williams VP, Bishop-Fitzpatrick L, Lane JD, Gwyther LP, Ballard EL, Vendittelli AP, et al. Video-based coping skills (VCS) to reduce health risk and improve psychological and physical wellbeing in Alzheimer's disease family caregivers. Psychosom Med. 2010;72:897-904.

31. Bruvik F, Ranhoff $A H$, Ulstein I, Engedal K. The effect of psychosocial support intervention on depression in PwAD and their family caregivers: an assessor-blinded RCT. Dement Geriatr Cogn Disord Extra. 2013;3:386-97.

32. Cooper C, Katona C, Orrell M, Livingston G. Coping strategies, anxiety and depression in caregivers of people with Alzheimer's disease. Int J Geriatr Psychiatry. 2008;23:929-36.

33. Tschanz BT, Deberard MS, Snyder C, Smith C, Lester Lee, Lyketsos CG. Caregiver coping strategies predict cognitive and functional decline in dementia: The Cache County Dementia Progression Study. Am J Geriatr Psychiatry. 2013;21:57-66.

\section{Correspondence:}

Alexandre Magno Frota Monteiro

Rua João Lira, 66

22430-210 - Rio de Janeiro, RJ - Brazil

Tel.: + 55 (21) 982046913

E-mail: alexandre@centronati.com 